\title{
What are the implications of accountable care organizations for physical medicine and rehabilitation practices?
}

\author{
John L. Melvin \\ Jefferson Medical College of Thomas Jefferson University \\ Greg Worsowicz \\ University of Missouri-Columbia
}

Follow this and additional works at: https://jdc.jefferson.edu/rmfp

Part of the Neurology Commons

Let us know how access to this document benefits you

\section{Recommended Citation}

Melvin, John L. and Worsowicz, Greg, "What are the implications of accountable care organizations for physical medicine and rehabilitation practices?" (2011). Department of Rehabilitation Medicine Faculty Papers. Paper 10.

https://jdc.jefferson.edu/rmfp/10

This Article is brought to you for free and open access by the Jefferson Digital Commons. The Jefferson Digital Commons is a service of Thomas Jefferson University's Center for Teaching and Learning (CTL). The Commons is a showcase for Jefferson books and journals, peer-reviewed scholarly publications, unique historical collections from the University archives, and teaching tools. The Jefferson Digital Commons allows researchers and interested readers anywhere in the world to learn about and keep up to date with Jefferson scholarship. This article has been accepted for inclusion in Department of Rehabilitation Medicine Faculty Papers by an authorized administrator of the Jefferson Digital Commons. For more information, please contact: JeffersonDigitalCommons@jefferson.edu. 


\title{
As submitted to:
}

\author{
$P M$ and $R$
}

\section{And later published as:}

\section{What Are the Implications of Accountable Care Organizations for PhysicalMedicine and Rehabilitation Practices?}

\author{
Volume 3, Issue 11, November 2011, Pages 1068-1071
}

\section{DOI: 10.1016/j.pmrj.2011.09.009}

John L. Melvin, MD, MMSc, Greg Worsowicz, MD, MBA

\section{Introduction}

The goal of delivering quality health care at a lower overall cost through accountable care organizations (ACO) as described as part of the Patient Protection and Affordable Care Act of 2010 (ACA) has gained momentum among payers, providers, and the public. The concept includes developing an organization that provides incentives for quality care while lowering the overall cost of the care. If the goals are met, then both providers and payers would be able to share in the financial savings produced by the lower health care costs achieved while improving the quality of care delivered. The Centers for Medicare and Medicaid Services (CMS) has further defined ACOs in the proposal rule released March 31, 2011, in the Medicare Shared Savings Program: Accountable Care Organizations. Medicare has projected that, over the first 3 years, there will be 75-150 ACOs developed, resulting in coverage of 1.5-4.0 million Medicare beneficiaries. This initial phase has been projected to save Medicare $\$ 510-\$ 800$ million in health care costs $[1,2]$. Regardless of whether or not this initial model of ACOs will have long-term validity, it is likely that components of the model will be included in future health care reform; therefore, physical medicine and rehabilitation (PM\&R) providers will have to decide how they will respond to these future changes. This article describes some of the CMS proposed ACO regulations and strategies that practices might consider to prepare for the future.

\section{Issues}

\section{The ACA, CMS, and ACOs}

Through the ACA, CMS provides funding for demonstration projects by using the ACO models that this legislation identifies $[1,3]$. CMS has published for comments proposed 
rules that designate the criteria for ACO demonstration projects [1]. The description of these models is somewhat generic because the authors of the ACA most likely wanted to explore the benefits and savings of ACOs developed by different stakeholders.

The goal of an ACO is to provide incentives for health care that focus on quality and efficiency rather than volume-based procedures. One approach is to establish systems that link payments to quality targets while reducing the total cost of care for defined patient populations. Anticipated cost reductions are expected from improved coordination of services and by linking these services to designated quality metrics. The participants of ACOs can include various health care providers, including physicians, hospitals, physician

assistants, nurse practitioners, and others [2]. Many of these rules have been published and outlined in previous publications $[2,4,5]$.

The CMS proposal includes requirements that an ACO

(A) have a minimum of 5000 patients, all of whom must be registered with primary care physicians,

(B) meet a minimum standard with respect to 65 quality measures before it is eligible for bonus payments,

(C) produce minimum saving targets based on the number of patients covered,

(D) must have a governing body composed of $75 \%$ ACO participants,

(E) have at least 1 patient sit on the governing board,

(F) cannot preclude at-risk beneficiaries to save money,

(G) must retain at least $25 \%$ of bonus payments as escrow for future losses $[1,2,5]$.

The criteria were disappointing to the limited number of health care providers who were in apparent positions to apply for ACO demonstration projects. Although there was wide acceptance of ACOs as described in the ACA, most provider groups have now indicated that, without changes in the current proposed rules, they will not apply to have demonstration projects. In fact, Modern Medicine (June 25, 2011) [2] reported that 93\% of American Medical Group Association members indicated that they would not participate in demonstration programs under the current proposed rules. Their concerns included the amount of capital necessary to begin an ACO, the risks of losing money, operational challenges of integrated systems, and the limited number of evidence-based treatment protocols that add to the difficulties in forming ACOs (Medical Economics, June 29, 2011) [5]. It is expected that each ACO will have to spend $\$ 1.75$ million to secure the support personnel and technology needed to participate $[4,6]$.

\section{Actual ACO Participation}


It may be unlikely that an individual PM\&R practice will have the opportunity to be an ACO participant in the near future because the requirements will limit the number of ACOs that develop. Individual PM\&R practices will need to determine if the option of participating in, or even if, an ACO will actually exist in their community (either within the next couple of years or at some time in the future). It is more likely that physiatrists who are part of group practices closely aligned with hospitals will have the first opportunities to participate in ACOs; however, they will do so only if regulations that govern ACOs have a reasonable balance between potential risks and rewards.

\section{Performance Metrics}

One basic principle of ACOs is the measurement of performance to assure that quality care is being provided. Some insurers independent of the ACO model already have developed pay-for-performance programs that reward practitioners for meeting specified quality targets; however, most of these programs do not include targets directly relevant to a PM\&R practice. Health Information Technology (HIT) legislation calls for all specialties to identify relevant existing quality targets or to develop targets for future use [7]. The National Quality Forum has endorsed a list of performance measures that are accepted by multiple organizations and regulatory agencies [8]. A review by the American Academy of Physical Medicine and Rehabilitation identified some of these National Quality Forum metrics that are relevant to PM\&R practices. It, therefore, is critical that practices monitor their local payers to determine whether they have relevant pay-for-performance programs and how practices might

participate in them.

\section{Commitment to Quality Measurement and Improvement}

All stakeholders anticipate that any future models of health care delivery will emphasize quality and safety standards. This emphasis will include outpatient care as well as the more established focus on inpatient care. Payers, regulators, and accrediting bodies will require the practices of all specialties

to record measures of quality and implement programs for its improvement. The Health Information Technology legislation initially provides bonus payments for meeting quality standards, but eventually will penalize practices that fail to meet these standards. At present, there are few specific standards for the conditions that PM\&R physicians treat so that their practices have some flexibility in the measures they use. Eventually, current efforts at developing quality standards are expected to result in specific standards for these conditions, and regulators are likely to mandate their use.

\section{ACO-related Revenue Expansion}


There may be opportunities for expanding outpatient PM\&R practice revenues through collaboration with models that incorporate some of the features of ACOs. One potential prospect is through patient-centered medical homes (PCMH), which are likely to be core components of ACOs. These are primary care practices that provide a "home" for patients and coordination of care, particularly for patients with chronic diseases. Some physiatric practices have arranged to provide part-time physiatrists to medical homes and provide services for patients with chronic diseases that primary care physicians feel less comfortable managing. Examples of this include managing patients with degenerative arthritis of the knees, chronic back pain, spinal cord injuries, and traumatic brain injuries, as well as participation in care coordination and postacute management of patients with disabilities. Primary care practices often vary regarding the conditions that they wish to refer to a specialist and their willingness to have a specialist spend time in their clinics. PM\&R practices should explore whether PCMH opportunities

exist locally.

\section{Implementing ACO Components}

\section{General.}

Many of CMS rules for ACOs include components that health care regulators and payers are likely to require in the future. These requirements will persist even if the CMS model of ACOs does not survive. PM\&R practices need to plan to include some of these components so as to position themselves for future developments, including participating in an ACO or preparing for changes in delivery models. Some basic decisions include making assumptions regarding who might be future partners in integrated delivery systems and who will have the responsibility for the health of identified patient populations.

\section{Electronic Medical Records.}

The PCMH standards currently require significant electronic technological support. These systems will need to

(A) disseminate critical patient data to the entire care team;

(B) allow patients to interact with care providers through

e-mail and Web portals, including accessing their medical records over the Internet;

(C) prescribe electronically;

(D) support quality measurement and performance improvement programs [6].

Medical PM\&R practices that hope to link to medical homes will need to acquire and use electronic medical records (EMR) capable of integrating into the medical home systems in which they participate. This could create dilemmas for some independent PM\&R 
practices because EMR systems vary in their capabilities and compatibilities. The choice of an EMR system may precede the selection of future health care system or provider partners, which makes it difficult to select the needed compatible EMR system.

Physiatrists in group practices or hospitals often use the EMRs of their partners and may not face this selection uncertainty. Although all medical practices eventually will need to use EMR systems, physiatrists who participate in medical homes and see patients at primary care practice sites may be able to

use the EMR and other support systems of those practices. This care strategy would permit PM\&R physicians to participate before they have implemented EMR systems, with all of the capabilities required for population-based health care.

\section{Adapting to Changing Payment Models}

The primary care model of PCMH aims at improving patient outcomes and providing less costly care by improving coordination of care. This requires additional revenue beyond the current fee-for-service system. Many payers independent of the ACO model accept the need for care management fees to provide the resources for this coordinated care. Much of this coordination can be through patients interacting with their care team by using their Web portals, nurse practitioners, care coordinators, or other physician extenders. Different payment models include per patient per month case management fees, enhanced fees for services, and/or incentive payments

for reporting quality measures. Some payers may pay a primary care practice for becoming a national committee for quality assurance (NCQA) recognized PCMH [9]. The senior author's (J.L.M.) PM\&R group partners with a general internal medicine practice that receives a supplement payment for care coordination. A major motivation for primary care practices to involve physiatrists in their medical homes would be to qualify for these supplemental forms of compensation for coordinated,

quality monitored care. Therefore, $P M \& R$ practices need to be flexible in how payment is received for their services. One approach is for physiatrists to collect their usual visit fees for physiatric services but receive the infrastructure support of the primary care practice either free or at favorable rates. If the primary care practice is at risk for its payments based upon meeting quality metrics, the participating PM\&R practice might share in the risk as well as the rewards. Physiatrists need to realize that health reform is likely to result in expansion of new compensation models and be prepared to participate in them. The ACO model involves negotiations among the various participating providers to allocate to each their share of episode of care payments (Table 1).

\section{Patient-Centered Care}

A major emphasis of the ACA is the principle of patientcentered care. The focus of this type of care is to respond to patient needs, not to the needs of providers. Many primary care practices used this principle before current specific models, such as PCMHs and 
ACOs. These models expect patients to be active participants in their care through open communication with their physicians and other providers.

This requires practices to be readily available to patients through visits, telephone calls, and electronic portals. Specialty practices linked to ACOs and PCMHs will need to provide similar levels of access to these patients. They also will need to develop communication links with the patients'

primary care providers. In collaboration with primary care practices, physiatrists have much to contribute to the patient-centered care of patients with chronic conditions. Physiatrists have expertise

in involving patients in their own care, working with the multiple professionals, integrating treatment plans and communicating information about these activities to all involved stakeholders. These physiatric skills of "patient centered care" and care coordination are the building blocks for a PCMH and, subsequently, for ACOs.

\section{Conclusion}

At present, it appears that the number of ACOs as envisioned in the ACA will be relatively limited unless CMS significantly alters the rules that they must follow. Initially, only a few PM\&R practices appear to have the opportunity of being ACO participants. However, many of the characteristics of ACOs are likely to exist in other health care delivery models. Depending on local conditions, practices may have current opportunities to enhance their revenues through participation in medical homes, pay-for-performance programs, or new models of care. All should explore how they will address future issues related to EMR systems, evolving payment systems, patient-centered care, and quality management. One thing appears certain, there is strong momentum for change in health care delivery. Is your practice prepared to position itself for these forthcoming changes?

\section{References}

1. Department of Health and Human Services. Available at www.ftc.gov/opp/aco /cmsproposedrule.pdf. Accessed July 26, 2011.

2. Boyle A. ACO Special Report: Under construction, under fire. Mod Med June 25, 2011. Available at http://www.modernmedicine.com/modernmedicine/Modern_Medicine_Now/ACOSpecial-ReportUnder-construction-underfire/ArticleStandard/Article/detail/728457?contextCategoryId_40748. Accessed July 25, 2011.

3. The Patient Protection and Affordable Care Act. Available at https://www.cms.gov/LegislativeUpdate/downloads/PPACA.pdf. Accessed July 21, 2011.

4. Mulvany C. Medicare ACOs no longer mythical creatures. Healthc Financ 
Manage 2011;65:96-104.

5. Lee KJ. Setting up a practical accountable care organization. Med Econ 2011:88;3438.

6. McBride M. The patient-centered house that technology built. Modern Med July 10, 2011. Available at http://www.modernmedicine.com/ modernmedicine/Modern_Medicine_Now/The-patient-centeredhouse- that-technologybuilt/ArticleStandard/Article/detail/731288?contextCategoryId_40748. Accessed July 25, 2011.

7. CMS Finalizes Definition of Meaningful Use of Certified Electronic Health Records (EHR) Technology files. Available at http://www.cms.gov/apps/media/press/factsheet.asp?Counter_3794\&intNumPerPage_10 \&checkDate_\&checkKey_\&srchType_1\&numDays_3500\&srchOpt_0\&srchData_\&key wordType_All\&chkNewsType_6\&intPage_\&showAll_\&pYear_\&year_\&desc_\&cboOrd er_date. Accessed July 27, 2011.

8. National Quality Forum. Available at http://www.qualityforum.org/Measures_List.aspx. Accessed July 25, 2011.

9. National Committee for Quality Assurance. Available at http://www.ncqa.org/tabid/631/Default.aspx. Accessed July 25, 2011. 
Table 1. Physiatric practice preparation for healith reform options

1. Monitor major payers for revenue enhancement options

a. Pay for performance
i. Meeting quality metrics
ii. $\theta$-prescribe/PQRS
iii. Meaningful use standards
iv. Individual payer opportunities
b. NCQA Recognition for PCMH

c. Centers of Excellence

2. Identify potential primary care practice collaborators

a. $\mathrm{PCMHs}$

b. PM\&R service in care management protocols

c. Other

3. Identify potential local ACO groups
a. Acadernic medical centers
b. Large group practices
c. Major hospitals
d. Payer groups

4. Explore options to participate in developing models
a. $\mathrm{PCMHs}$
b. ACOs
c. Other

5. Initiate quality program

a. Identify measures for high volume patients with chronic conditions

b. Monitor development of consensus measures for these conditions

c. Select meaningful use metrics

6. Implement EHR

7. Develop flexible payment models for services

a. Fee for service
i. Reduce overhead costs
ii. Sharing of infrastructure
iii. Integrated EHR

b. Risk sharing

i. Profit of care model

ii. Shared savings programs

8. Monitor regulatory and legal environment

PQRS = Physician Quality Reporting System; PCMH = potient-centered medical homes; $\mathrm{NCQA}=$ national committee for quality assurance; PM\&R = physical medicine and rehabilitation; $\mathrm{ACO}=$ accountable care organizations; EHR $=$ electronic health record 